\title{
THE EFFECT OF REINNERVATION ON COLLAGEN FORMATION IN DEGENERATING SCIATIC NERVES OF RABBITS
}

\author{
BY \\ M. ABERCROMBIE and M. L. JOHNSON \\ From the Department of Anatomy, University College, London
}

(RECEIVED 22ND MARCH, 1947)

\begin{abstract}
ONE of the phenomena of Wallerian degeneration in peripheral nerves is the formation of new endoneurial collagen. In a previous paper (Abercrombie and Johnson, 1946) we estimated the amount of collagen formed in degenerating sciatic nerves of rabbits during various periods up to two hundred days after the nerves had been severed. We found that significant amounts of collagen are formed during all periods of degeneration studied. The purpose of the investigation reported in the present paper is to determine whether reinnervation has any effect on this process of collagen formation. To that end we have compared the collagen content of completely severed nerves which undergo degeneration in the absence of reinnervation (these are referred to as non-reinnervated nerves), with the collagen content of crushed nerves which undergo degeneration but are very rapidly reinnervated (these are referred to as reinnervated nerves). One hundred days were allowed to elapse after the severance or crush before the nerves were removed for collagen estimation; a period during which an easily detectable amount of collagen is formed in normal degeneration.
\end{abstract}

\section{Method}

Our material consisted of the sciatic nerves of twelve adult rabbits, each providing one reinnervated and one non-reinnervated nerve. In each rabbit the sciatic nerve of one side was cut $1.5 \mathrm{~cm}$. below the level of the third trochanter, and the central stump was sutured to the skin. The result of this procedure is Wallerian degeneration throughout the peripheral stump with little or no reinvasion by axons. The sciatic nerve on the other side of each rabbit was crushed with smoothtipped forceps, also at $1.5 \mathrm{~cm}$. below the level of the third trochanter. The effect of crushing in this way is to interrupt all nerve fibres (axonotmesis), and this is followed by rapid and complete reinnervation. The new. axons enter the degenerated peripheral zone after a latent period averaging five days, and they extend throughout the region of nerve studied within about twenty-five days of the injury (Gutmann and others, 1942), and thereafter increase in diameter, reaching approximately one third of their normal volume at one hundred days, and their normal volume at about two hundred and fifty to three hundred days (Gutmann and Sanders, 1943).

One hundred days after the initial operation each animal was killed and part of each of its sciatic nerves taken for collagen estimation. The part of each nerve used for estimation consisted of the whole sciatic nerve in the thigh and knee peripheral to a point one-half $\mathrm{cm}$. below the original crush or cut; together with the anterior and posterior tibial branches in the shank down to a level $3 \mathrm{~cm}$. below the point of origin of the branch of the peroneal to the extensors of the shank.

After removal of the epineurium, the collagen content of the twenty-four nerves was determined simultaneously and under identical conditions by the method of Lowry and others (1941), and slightly modified as detailed in our previous paper (Abercrombie and Johnson, 1947). As in the experiments recorded in that paper, half the nerves were extracted in $\mathrm{NaOH}$ for twenty-four hours, and half for forty-eight hours.

For each nerve we obtained, as in our previous paper, estimates of the nitrogen content of the initial $\mathrm{NaOH}$ extract ("NaOH-soluble $\mathrm{N}$ "); of the supernatant after autoclaving (" collagen $\mathbf{N}$ ") ; and of the residue after autoclaving (" residue $\mathrm{N}$ ").

The figures given in the Table are means of the absolute values for the standard length of nerve removed; and means of " pair-differences," a pair-difference being the value for the reinnervated nerve minus the value for the non-reinnervated nerve of each rabbit taken individually. It should be noted that in our previous paper we did not express the results of our analyses in absolute values, but in a standardized form. Since in that work each rabbit provided an undegenerated sciatic nerve as well as a degenerated one, we were able to express the estimates for degenerated nerve as $\mathrm{mg}$. $\mathrm{N}$ per $100 \mathrm{~g}$. wet weight of the equivalent length of undegenerated nerve, which is obviously not possible in the present work. 
TABle

\begin{tabular}{|c|c|c|c|c|c|}
\hline & Wet weight & Collagen $\mathrm{N}$ & $\underset{N}{\mathrm{NaOH}-\text { soluble }}$ & Residue $\mathbf{N}$ & 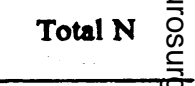 \\
\hline Non-reinnervated nerves .. & $93 \pm 5$ & $0.82 \pm 0.05$ & $1.21 \pm 0.05$ & $0.014 \pm 0.002$ & $2.04 \pm 0.10 \mathrm{C}$ \\
\hline Reinnervated nerves $\quad$. & $115 \pm 5$ & $0.87 \pm 0.04$ & $0.45 \pm 0.04$ & $0.047 \pm 0.004$ & \\
\hline $\begin{array}{ccc}\text { Gain by reinnervation (pair- } \\
\text { differences) } & . . & . .\end{array}$ & $23 \pm 6$ & $0.05 \pm 0.04$ & $0.24 \pm 0.06$ & $0.032 \pm 0.004$ & $0.33 \pm 0.10 \stackrel{\stackrel{m}{\rightleftarrows}}{\stackrel{\text { f }}{\rightleftarrows}}$ \\
\hline Undegenerated nerves $\quad$. & $82 \pm 2$ & $0.47 \pm 0.01$ & $0.92 \pm 0.02$ & $0.17 \pm 0.01$ & $1.56 \pm 0.03 \stackrel{\frac{\bar{c}}{+}}{\frac{\sigma}{C}}$ \\
\hline
\end{tabular}

Mean values in mg. per standard length of nerve with standard errors

The proximal half-centimetre of the non-reinnervated sciatic nerve stump, and the posterior tibial nerve of the reinnervated sciatic distal to the region used for collagen analysis, were fixed and stained by Bodian's method for axons. Most of the non-reinnervated stumps were devoid of axons, though there were a few stray ones in three of them. All the reinnervated stumps contained numerous well-developed axons.

As a check on the consistency of our method of estimation, it is important to compare the values obtained for the set of 100-day non-reinnervated nerves in this experiment with the values independently obtained for similar nerves reported in our previous paper (Abercrombie and Johnson, 1946). The analyses recorded in the present paper were done in one simultaneous batch, while those recorded in the previous paper were made at several different times. In the previous paper, as stated above, the $\mathrm{N}$ contents and wet weights were expressed in a different way from that used here. But since on the average the same amount of nerve was removed from each rabbit in the two experiments, the mean absolute values (not published in the previous paper) can be directly compared. That we were successful in removing a well-standardized amount of nerve is shown by the fact that mean wet weight and total $\mathbf{N}$ content do not differ, within our limits of accuracy, between the two sets of 100-day degenerated nerves. The collagen $\mathrm{N}$ is rather lower, and the $\mathrm{NaOH}$-soluble $\mathbf{N}$ rather higher in the earlier set of nerves, but these differences are not significant $(P=0.1$ in both). The residue $\mathrm{N}$, however, is almost twice as high in the earlier set of nerves, and the difference is significant $(P=<0.01)$. The consistency of the method is, therefore, satisfactory except in the case of the residue $N$, which is a very small fraction of the total $\mathrm{N}$. Consistency of estimates of residue $\mathrm{N}$ within a single batch of simultaneous analyses is good, as the standard errors in the Table show; but different batches differ rather disconcertingly from each other.

\section{Results}

In the Table we give the mean wet weight of the standard length of nerve analysed, and the mean $\mathbf{N}$ content of each of the different fractions investigated, with their respective standard errors. The final column, "Total $\mathrm{N}$ ", is the sum of the three presceding columns, and, as explained in the previous paper, falls a little short of the real total $N$ sinee lipin $\mathbf{N}$ is only partly estimated. In the upper row is the mean value of the twelve non-reinnervatof nerves; and in the second row that of the twelve reinnervated nerves. The third row gives the mean of the pair-differences, each pair-difference being obtained by subtracting, for each pair of nerves, the non-reinnervated value from the reinnervated value. The fourth row gives for comparison mean values for thirty-three normal undegenerated nerves (from data used, in a different form, by Abercroribie and Johnson, 1946). The mean value for the reinnervated nerves is higher than that for the reinnervated nerves, in every column of the Tabli The pair-differences for wet weight of nexive, $\mathrm{NaOH}$-soluble $\mathbf{N}$ content, residue $\mathbf{N}$ content and total $\mathrm{N}$ content, are significantly different from zero by $t$ test $(P=<0.01$ in each case). The dif ference in collagen content is, however, quile insignificant $(\mathrm{P}=0 \cdot 25)$.

\section{Discussion}

In our previous paper (Abercrombie and Johnson 1946) we showed that significant amounts of collage are formed in the sciatic nerve during the first fifty days of degeneration and between fifty and hundred days. Some collagen is probably also formed during the first twenty-five days. The indications are that collagen formation goes on continuously throughout the first hundred days of degeneration (excluding probably the first few days after severance, when the phenomena of degeneras. tion have hardly appeared); and, once started the rate of formation does not seem to change greatly during this period. We have now found that one hundred days after division a nerve hăs the same collagen content whether or not it has been reinnervated.

We presume, therefore, that the whole process 
of collagen formation proceeds, regardless of whether reinnervation takes place or not, for at least the first hundred days after division of the nerve. Collagen formation during Wallerian degeneration when reinnervation is prevented continues, of course, well beyond one hundred days (Abercrombie and Johnson, 1946), and we cannot deny that beyond a hundred days reinnervation may modify the process of fibrosis. But the surprising absence of any demonstrable effect during the first hundred days suggests that a later effect is not likely to be important.

The other components $(\mathrm{NaOH}$-soluble $\mathrm{N}$, residue $\mathrm{N}$, and also wet weight), investigated incidentally to the determination of collagen content, are distinctly different in the reinnervated and nonreinnervated nerves. It is not that there is a general tendency for the composition of a reinnervated nerve to approach that of an undegenerated nerve (see Table). It seems to be rather that a reinnervated nerve represents, in weight and chemical composition, a degenerated nerve with new nervefibres superimposed. If further work can establish this, the way is open to a quantitative analysis of the growth of new axons during the process of reinnervation. Assuming the point for the present, the pair-differences in the Table represent quantities of nerve-fibre material formed during one hundred days in the particular length of nerve used. It appears that the new nerve-fibre material has a lower $\mathbf{N}$ content relative to its wet weight than the rest of the nerve (1.4 per cent. of wet weight for the nerve-fibres, against $2 \cdot 2$ per cent. for the nonreinnervated nerve), but this conclusion is, of course, tentative.

As to the vexed question whether Schwann cells or endoneurial fibroblasts are responsible for collagen formation in peripheral nerves, our results give no conclusive answer. But they might be taken to give a little support to the view that endoneurial fibroblasts are implicated. Reinnervation involves the development of an intimate association between Schwann cells and axons. In some respects this is known to affect the Schwann cells profoundly, switching them on to myelin production (see Speidel, 1933) and inhibiting their powers of migration in tissue culture (Abercrombie, Johnson, and Thomas, unpublished). The endoneurial cells, on the other hand, do not come into contact with the new axons, and they are not known to be affected by reinnervation in any way. The absence of an effect of reinnervation on collagen formation, therefore, parallels the absence of any known effect on endoneurial cells, but does not parallel the existence of a profound effect on Schwann cells.
It should be noted that there is no sign of the fibrosis-inhibiting substance which Weiss (1943) has suggested diffuses centrifugally along a nerve. The crushed nerve, which is left in continuity with the undamaged central stump and with the central nervous system, would, according to Weiss's suggestion, be expected to undergo some inhibition of fibrosis in comparison with the cut nerve, unless the crushed region prevents the centrifugal flow entirely, which seems unlikely. In fact there is no demonstrable inhibition of fibrosis in the crushed nerve.

Holmes and Young (1942) and Sanders and Young (1944) found that during prolonged degeneration the Schwann tubes of the peripheral stump of a severed nerve shrink considerably ; and that when such a stump becomes reinnervated, the shrunken tubes fail to expand again to their original size. They suggested that the collagen formed in a peripheral stump during its degeneration might be responsible for limiting re-expansion. On the other hand early reinnervation, after a brief period of degeneration, leads to the reconstitution of a much more normal tube size (Sanders and Young, 1944). Although it seems entirely reasonable to suppose that new collagen prevents expansion after late reinnervation, the larger tube expansion which follows early reinnervation is not easy to explain. It is clear from our results that the difference in ultimate tube size according to time of reinnervation is not due to any inhibition of collagen formation by reinnervation. The difference is probably due to the outward pressure of the growing axons being exerted, in the case of late reinnervation after most of the collagen has been formed, and in the case of early reinnervation almost throughout the period of collagen formation. When the latter happens, the early expansion is opposed by only small amounts of new collagen ; and when more collagen has formed the tubes have already partly expanded and need to stretch the more recently formed collagen relatively little in order to reach normal tube diameter.

\section{Summary}

Estimates have been made, by a chemical method, of the collagen content of rabbit sciatic nerves peripheral to a point where their nerve fibres had been experimentally interrupted a hundred days earlier in one of two different ways : (a) by complete severance of the nerve, preventing reinnervation, (b) by crushing, permitting rapid reinnervation.

There was no significant difference in collagen content between the reinnervated and the 
non-reinnervated nerves. The process of collagen formation set going by interrupting a nerve is apparently uninfluenced by reinnervation.

Wet weight and non-collagen $\mathbf{N}$ were higher in the reinnervated nerves, the difference probably representing approximately the material of the new nerve fibres.

The experimental work recorded here was done in the Department of Zoology, University of Birmingham. Part of the expenses was deferred by a grant from the Medical Research Council. We should like to thank Miss Kathleen Rice for her valuable technical assistance ; and Prof. J. Z. Young for criticizing the manuscript.
REFERBNCES

Abercrombie, M., and Johnson, M. L. (1946). Neurol. Neurosurg. Psychiat., 9, 113.

Gutmann, E., Guttman, L., Medawar., P. B., and Younge J. Z. (1942). J. exp. Biol., 19, 14. Gutmann, E., and Sanders, F. K. (1943). J. Physiol :ु

Holmes, W., and Young, J. Z. (1942). J. Anat. Lond. $77,63$.

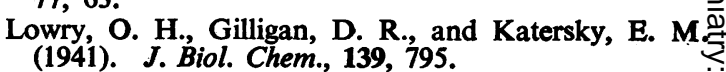

Sanders, F. K., and Young, J. Z. (1944). J. Physiol. $\vec{\Rightarrow}$ 103, 119.

Speidel, C. C. (1933). Amer. J. Anat., 52, 1. Weiss, P. (1943). Anat. Rec., 86, 491. 